\section{GENRE} en séries

\section{Genre en séries}

Cinéma, télévision, médias

$6 \mid 2017$

Être mère, être père : représentations et discours médiatiques

\title{
Marlène Coulomb-Gully, Huit femmes sur un plateau. Télévision, journalisme et politique
}

Paris, Nouveau Monde Éditions, 2016, 232 pages

\section{Rayya Roumanos}

\section{(Q) OpenEdition \\ Journals}

\section{Édition électronique}

URL : http://journals.openedition.org/ges/1010

DOI : $10.4000 /$ ges. 1010

ISSN : 2431-6563

\section{Éditeur}

Presses universitaires de Bordeaux

\section{Référence électronique}

Rayya Roumanos, « Marlène Coulomb-Gully, Huit femmes sur un plateau. Télévision, journalisme et politique », Genre en séries [En ligne], 6 | 2017, mis en ligne le 01 novembre 2017, consulté le 18 février 2021. URL : http://journals.openedition.org/ges/1010 ; DOI : https://doi.org/10.4000/ges.1010

Ce document a été généré automatiquement le 18 février 2021.

\section{(c) (i) ()}

La revue Genre en séries est mise à disposition selon les termes de la Licence Creative Commons Attribution - Pas d'Utilisation Commerciale - Pas de Modification 4.0 International. 


\section{Marlène Coulomb-Gully, Huit femmes sur un plateau. Télévision, journalisme et politique}

Paris, Nouveau Monde Éditions, 2016, 232 pages

\section{Rayya Roumanos}

\section{RÉFÉRENCE}

Marlène Coulomb-Gully, Huit femmes sur un plateau. Télévision, journalisme et politique, Paris, Nouveau Monde Éditions, 2016, 232 pages

1 Le titre, polysémique, interpelle, la couverture, élégante, invite à feuilleter avec attention l'ouvrage et le résumé, déconcertant et incisif, convainc même les plus sceptiques de s'abandonner à la lecture de ce texte puissant : 8 portraits de femmes qui ont réussi à s'imposer comme journalistes politiques de télévision, dans un univers professionnel dominé par les hommes.

2 Pour un lecteur averti, c'est le nom de l'auteure qui constitue la première accroche, l'assurance d'une lecture à la fois agréable et exigeante. Marlène Coulomb-Gully est spécialiste du rapport entre médias, genre et politique, thème qu'elle déconstruit sous différents angles dans de nombreux ouvrages et articles scientifiques inscrits dans le champ des sciences de l'information et de la communication. Professeure à l'université Toulouse 2-Jean Jaurès, elle travaille au sein du Laboratoire d'études et de recherches appliquées en sciences sociales (LERASS) sur les rapports sociaux de sexe et sur les représentations des hommes et des femmes dans les médias. Son dernier ouvrage s'inscrit logiquement dans la continuité de ces travaux de recherche même s'il suit une démarche sensiblement différente; ici, il ne s'agit pas, à proprement parler, d'une recherche académique sur les trajectoires professionnelles de femmes qui ont brillé dans le domaine du journalisme politique, mais d'une galerie de portraits qui puise sa force dans une approche plus journalistique qu'empirique. 
3 L'ouvrage est composé de huit chapitres, un par journaliste, des pionnières Danièle Breem, Jacqueline Baudrier et Michèle Cotta aux continuatrices Arlette Chabot, Ruth Elkrief et Audrey Pulvar en passant par les stars Christine Ockrent et Anne Sinclair. L'introduction, courte, expose le double objet du recueil : raconter le parcours de huit femmes brillantes dans l'exercice d'un métier longtemps considéré comme une exclusivité masculine et, à travers elles, retracer l'histoire de la télévision française.

Pris dans son ensemble, le récit est un voyage à travers les âges, ceux du petit écran comme ceux de la société française. Le lecteur ne peut s'empêcher de noter à quel point certaines histoires sont ancrées dans leur époque et certaines réactions datées, comme cette incroyable anecdote relatée dès l'ouverture du livre sur Danièle Breem obligée de subir, sans sourciller, le geste insensé de ses collègues masculins qui lui pissaient sur les pieds pendant qu'elle lisait ses textes à l'antenne (p.9), ou encore cette remarque dédaigneuse qu'aurait adressé un des collègues de Jacqueline Baudrier au patron des programmes de l'ORTF : « quoi ? Le journal est fait par une gonzesse ?» (p. 42)

Pourtant, à y regarder de plus près, et c'est là le propos de l'ouvrage, peu de choses ont véritablement changé pour les femmes en soixante-dix années d'histoire de la télévision. Alors même qu'elles ont largement contribué à faire gagner à ce médium ses lettres de noblesse face à la presse écrite détentrice de la légitimité journalistique, les femmes doivent, aujourd'hui encore, déjouer les pièges de la ségrégation horizontale (l'orientation des femmes vers des métiers "conformes » à leur sexe) et verticale (le plafond de verre qui les écarte de la gouvernance) pour gravir les échelons et compter parmi les acteurs clés du secteur télévisuel. Car, comme le rappelle judicieusement l'auteure, «si l'égalité de droit est un fait, l'égalité de fait reste bien souvent un droit encore à conquérir.» (p. 222)

6 Le récit de Marlène Coulomb Gully revient sur le combat de huit femmes différentes tant au niveau de leur caractère qu'au niveau de leurs engagements sociaux et politiques et de leur style journalistique. Si toutes ont cherché à dépasser les rôles qui leur ont été assignés, certaines ont réussi à surmonter les injonctions sociales faites à leur sexe dans une perspective féministe quand d'autres ont inscrit leur combat dans une logique plus individualiste et néanmoins bénéfique à l'avancement du statut des femmes journalistes. Pour faire entendre leur voix dans l'univers du journalisme politique, les pionnières ont dû décloisonner un champ dominé par l'image d'une sociabilité masculine plus favorable à la proximité entre journalistes et hommes politiques du même sexe, au moment où des professionnelles comme Christine Ockrent, Anne Sinclair ou encore Audrey Pulvar, ont dû repousser des procès d'intention liés à leur prétention salariale ou aux fonctions politiques de leur conjoint.

7 Marlène Coulomb-Gully reconstruit leurs histoires intimes et professionnelles à la manière d'une journaliste qui dresse le portrait de personnalités publiques grâce à un long travail de documentation, de contextualisation et de récupération d'anecdotes sur la vie des protagonistes de son récit. Cette tâche, souvent difficile, a nécessité, pour certaines d'entre elles (celles d'avant la "pipolisation », note l'auteure) de reconstituer le puzzle des premières années et pour d'autres de sélectionner, parmi la profusion d'informations qui les concernent, celles qui restent fidèles à la réalité.

Quand cela s'est avéré possible, l'auteure est allée à leur rencontre pour recueillir leur témoignage et déterminer le sens qu'elles donnent à leur parcours ainsi que l'interprétation qu'elles font des situations conflictuelles qu'elles ont dû traverser. Pour construire son récit, elle ne s'est pas contentée de consulter les ouvrages savants sur 
l'univers de la télévision ou les monographies consacrées à ces grandes dames du petit écran, mais a également interrogé plusieurs acteurs et actrices de ce champ et s'est appuyée sur des documents audiovisuels de l'INA et sur la presse généraliste et spécialisée pour reconstituer leurs trajectoires aussi bien personnelles que médiatiques. Ses nombreux travaux académiques sur le triptyque media/genre/ politique ont également nourri son ouvrage offrant des explications claires et perspicaces aux choix des protagonistes et aux réactions publiques et professionnelles qui les ont accompagnées.

Plus qu'un récit journalistique dont l'unique visée serait de rattacher l'histoire de ces femmes à l'actualité, les différents chapitres révèlent les analogies et les correspondances entre leur trajectoire afin d'identifier les manifestations sexistes qui entravent l'avancée des femmes journalistes sur plusieurs décennies. L'approche journalistique choisie par l'auteure agit, en réalité, comme un outil de démocratisation d'une réflexion complexe sur les rapports de domination banals et intériorisés par tout un chacun, bien au-delà du secteur télévisuel, et comme un générateur de questionnements nécessaires sur les conditions professionnelles et sociales des femmes dans un système toujours inégalitaire.

Cet ouvrage d'une lecture fluide et agréable est avant tout un texte grand public. Pour autant, en refermant le recueil, le spécialiste ne ressentira pas la frustration d'une réflexion inaboutie ou d'une analyse incomplète. Il appréciera, au contraire, ce parti pris d'incarner une problématique complexe dans des histoires aussi singulières qu'exemplaires.

\section{AUTEURS}

\section{RAYYA ROUMANOS}

Rayya Roumanos est maître de conférences à l'Institut de journalisme de Bordeaux Aquitaine (IJBA) - Université Bordeaux Montaigne et membre du laboratoire MICA EA 4426. Ses recherches portent sur le rapport entre média et société dans le monde contemporain avec une attention particulière aux dimensions culturelles et identitaires de l'information et de la communication à l'heure de la globalisation. 